LA-UR- 09-05052

Approved for public release;

distribution is unlimited.
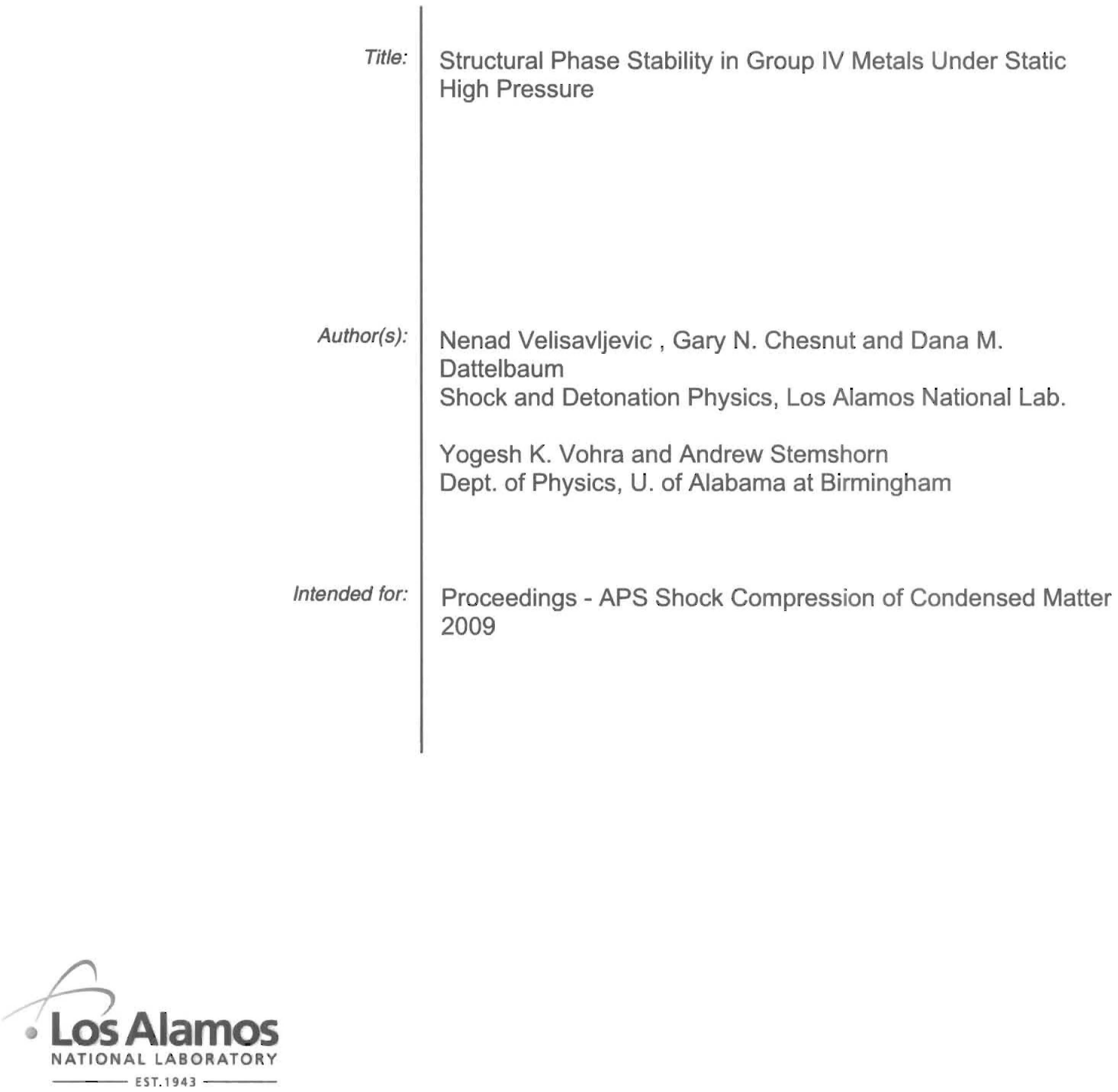

Los Alamos National Laboratory, an affirmative action/equal opportunity employer, is operated by the Los Alamos National Security, LLC for the National Nuclear Security Administration of the U.S. Department of Energy under contract DE-AC52-06NA25396. By acceptance of this article, the publisher recognizes that the U.S. Government retains a nonexclusive, royalty-free license to publish or reproduce the published form of this contribution, or to allow others to do so, for U.S. Government purposes. Los Alamos National Laboratory requests that the publisher identify this article as work performed under the auspices of the U.S. Department of Energy. Los Alamos National Laboratory strongly supports academic freedom and a researcher's right to publish; as an institution, however, the Laboratory does not endorse the viewpoint of a publication or guarantee its technical correctness.

Form $836(7 / 06)$ 


\title{
STRUCTURAL PHASE STABILITY IN GROUP IV METALS UNDER STATIC HIGH PRESSURE
}

\author{
N. Velisavljevic ${ }^{1}$, G. N. Chesnut ${ }^{1}$, D. M. Dattelbaum ${ }^{1}$, Y. K. Vohra ${ }^{2}$, and A. Stemshorn ${ }^{2}$

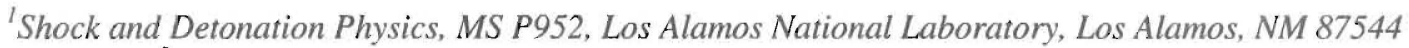 \\ ${ }^{2}$ Physics Dept., University of Alabama at Birmingham, Birmingham, AL 35294
}

\begin{abstract}
In group IV metals ( $\mathrm{Ti}, \mathrm{Zr}$, and $\mathrm{Hf}$ ) room temperature compression leads to a martensitic transformation from a ductile $\alpha$ to a brittle $\omega$ phase. $\alpha \rightarrow \omega$ phase boundary decreases to lower pressure at high temperature and can limit the use of group IV metals in industrial applications. There is a large discrepancy in the transition pressure reported in literature, with some of the variation attributed to experimental conditions (i.e. hydrostatic vs. non-hydrostatic). Shear deformation in non-hydrostatic experiments drives $\alpha \rightarrow \omega$ transition and decreases transition pressure. Impurities can also aid or suppress $\alpha \rightarrow \omega$ transition. By performing x-ray diffraction experiments on samples in a diamond anvil cell we show that interstitial impurities, such as $\mathrm{C}, \mathrm{N}$, and $\mathrm{O}$ can obstruct $\alpha \rightarrow \omega$ transition and stabilize $\alpha$ phase to higher pressure. We also show that reduction in grain size can also influence $\alpha-\omega$ phase boundary and help stabilize $\alpha$ phase to higher pressure under non-hydrostatic conditions.
\end{abstract}

Keywords: Group IV metals, high pressure, diamond anvil cell.

PACS: 61.05.cp, 61.50.Ks.

\section{INTRODUCTION}

Group IV metals titanium (Ti), zirconium (Zr), and hafnium (Hf), are characterized by a partially filled $3 \mathrm{~d}, 4 \mathrm{~d}$, and $5 \mathrm{~d}$ band respectively. A relative increase in electron number of the d-band, resulting from $\mathrm{s} \rightarrow \mathrm{d}$ electron transfer, is expected under applied pressure [1]. At ambient conditions $\mathrm{Ti}, \mathrm{Zr}$, and Hf crystallize in hexagonal close packed structure (hcp, $\alpha$ ). The pressure driven increase in the number of d-electrons is believed to drive a structural phase transition in group IV metals toward a more energetically favorable high pressure body center cubic (bcc, $\beta$ ) crystallographic phase. In both $\mathrm{Zr}$ and $\mathrm{Hf}$ the structural phase transition from $\alpha$ to $\beta$ phase via an intermediate hexagonal $(\omega)$ phase, with three atoms per unit cell, has been observed [2]. Likewise, the $\alpha \rightarrow \omega$ structural phase transition has also been observed in Ti. A martensitic transformation from a ductile $\alpha$ to a brittle $\omega$ phase is observed at $\sim 8 \mathrm{GPa}$ for $\mathrm{Ti}$, $\sim 8 \mathrm{GPa}$ for $\mathrm{Zr}$, and $\sim 38 \mathrm{GPa}$ for Hf. The $\alpha \rightarrow \omega$ phase boundary decreases to lower pressures at high temperatures [3] and can severely limit the use of group IV metals in industrial applications.

Since the driving force for the $\alpha \rightarrow \omega$ structural phase transition is a pressure-driven increase in number of d-band electrons, the phase boundary can be strongly dependant on the presence of substitutional impurities. Substitutional impurities such as $\mathrm{Al}, \mathrm{V}, \mathrm{Mo}$, etc., can either increase or decrease the number of d-band electrons [4]. In the case of commercially available Ti-6Al-4V, alloying $\mathrm{Ti}$ with $\mathrm{Al}$, which effectively reduces the number of d-band electrons by two and $\mathrm{V}$, which increases $\mathrm{d}$-band concentration by one, leads to a shift in $\alpha \rightarrow \omega$ phase boundary at room temperature to $27 \mathrm{GPa}$ [5]. Likewise, interstitial impurities are also expected to have a significant affect on the $\alpha \rightarrow \omega$ phase boundary. In the case of interstitial 
impurities, the results of $A b$ initio calculations show that oxygen, nitrogen and carbon, which occupy the hexahedral and octahedral sites, increase the energy barrier and block the $\alpha \rightarrow \omega$ transformation [6].

The high susceptibility of the $\alpha \rightarrow \omega$ phase boundary to be influenced by impurities, both substitutional and interstitial, as well as possibly other factors such as grain size, texturing, etc., has lead to a large discrepancy in the reported transition pressure. In case of $\mathrm{Ti}$, for example, reported transition pressure values have ranged from $3 \mathrm{GPa}$ up to $11 \mathrm{GPa}$ [7]. Even when multiple experiments are performed on the same sample, the $\alpha \rightarrow \omega$ transition pressure can vary due to the effects of experimental conditions, i.e. compression under non-hydrostatic conditions leads to shear deformation which drives the structural phase transition and lowers the $\alpha \rightarrow \omega$ transition pressure [7].

In this article we report initial results of high pressure experiments performed on $\mathrm{Zr}$ and $\mathrm{Ti}$. Our results provide experimental evidence for the effects of interstitial impurities and grain size on the $\alpha \rightarrow \omega$ phase boundary. We also discuss some of the other factors that can lead to discrepancy in determining the onset of $\alpha \rightarrow \omega$ structural phase transition.

\section{EXPERIMENTAL PROCEDURE}

Three $\mathrm{Zr}$ samples, with varying interstitial impurity concentration, as shown in Table 1 , and Ti with a $\sim 100 \mathrm{~nm}$ grain size were investigated under static high pressures, using DACs. Both angle dispersive $\mathrm{x}$-ray diffraction (ADXD) and electrical resistance measurement techniques were utilized to investigate the onset of $\alpha$ to $\omega$ structural phase transition.

TABLE 1. Interstitial impurity concentration (Wt ppm) of three $\mathrm{Zr}$ metal samples investigated.

\begin{tabular}{llll}
\hline Sample & $\mathbf{O}$ & $\mathbf{N}$ & $\mathbf{C}$ \\
\hline Zr I & $<50$ & $<20$ & 22 \\
Zr II & 390 & 15 & 70 \\
Zr III & 1,200 & 80 & 270 \\
\hline
\end{tabular}

\section{ADXD experiments}

For ADXD experiments, the metal sample and $\mathrm{Cu}$ pressure marker were loaded in a pre-indented spring steel gasket with a 90 micron sample hole. $\mathrm{ADXD}$ experiments were performed on samples under both quasi-hydrostatic, using methanolethanol (4:1) mixture, and non-hydrostatic pressure conditions. Beamlines $16 \mathrm{ID}-\mathrm{B}$ and BM-D at high pressure collaborative access team (HPCAT), Advanced Photon Source (APS), at Argonne National Laboratory were used to perform $\mathrm{x}$-ray diffraction experiments. Prior to measurements, the sample to detector distance was calibrated using a $\mathrm{CeO}_{2}$ calibration standard.

\section{Electrical resistance measurements}

Designer diamond anvils [8] were used in the DAC in order to measure the electrical resistance of the sample as a function of applied pressure. In order to establish good electrical contact between the probes and the sample, pressure media were not used and all measurements were performed under non-hydrostatic conditions. Furthermore, in order to avoid possible interference from $\mathrm{Cu}$, we opted to use ruby fluorescence technique for determining pressure. Since the electrical probes in designer anvil are only exposed near the center of the diamond, where contact is made with the sample, and elsewhere they are insulated within single crystalline diamond layer, all samples were loaded in pre-indented spring steel gasket. All measurements were made using the Van der Pauw four-probe technique in order to eliminate the resistance of the probes and help detect small changes in our metallic sample.

\section{RESULTS AND DISCUSSION}

Initial ADXD spectra collected at room temperature and pressure conditions shows that all three $\mathrm{Zr}$ samples and $\mathrm{Ti}$ sample are in $\alpha$ phase. Ambient condition lattice parameter values $\mathrm{a}_{0}=$ $3.232 \pm 0.002 \AA$ and $c_{0}=5.146 \pm 0.002 \AA(\mathrm{c} / \mathrm{a}=$ $1.593)$ and volume/atom $=23.270 \AA^{3}$ were determined for $\mathrm{Zr}$ samples, while $\mathrm{a}_{0}=2.949 \pm 0.002$ $\AA$ and $\mathrm{c}_{0}=4.689 \pm 0.002 \AA(\mathrm{c} / \mathrm{a}=1.590)$ and 
volume/atom $=17.658 \AA^{3}$ was measured for $\mathrm{Ti}$. Under applied pressure, and with both hydrostatic and non-hydrostatic conditions, all samples underwent a structural phase transition from $\alpha$ to $\omega$. The onset of $\alpha \rightarrow \omega$ transition, in all cases, can most easily be identified by the appearance of (001) and $(110 / 101) \omega$ phase peaks, which grow in at the expense of decreasing (100), (002), and (101) $\alpha$ phase peaks, as shown in Fig. 1. In all of our measurements, once the $\alpha \rightarrow \omega$ transition was observed to initiate, both phases co-exist over a certain pressure range.

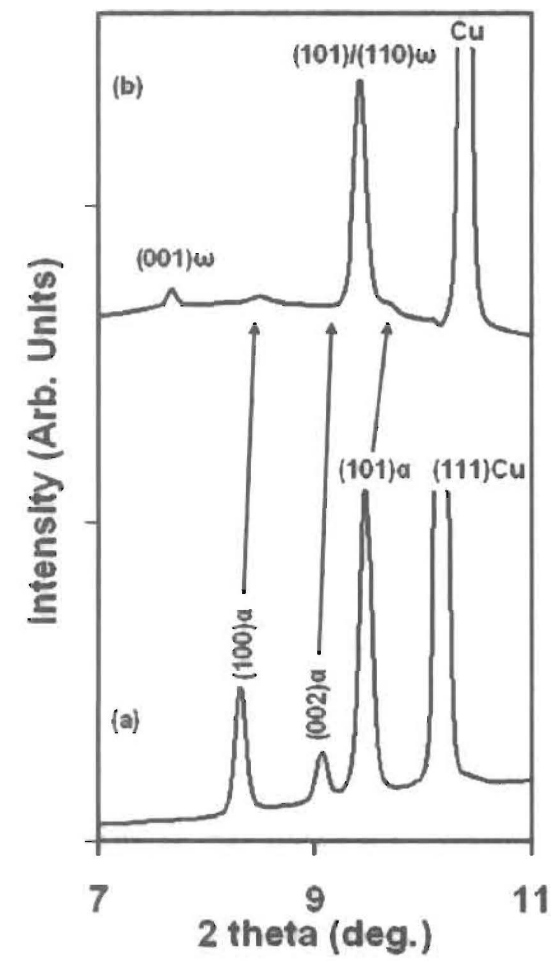

Figure 1. Typical ADXD spectra collected for $\mathrm{Zr}$ and $\mathrm{Ti}$ sample. Shown here is spectra collected for Ti - initially the sample is comprised on only $\alpha$ phase (a), once pressure is increased above certain value onset of $\alpha \rightarrow \omega$ structural phase transition is observed (b).

For experiments performed on $\mathrm{Zr}$ samples, we observed that the pressure of the $\alpha \rightarrow \omega$ transition increased with increasing interstitial impurity concentration. For example, under hydrostatic conditions, preliminary data shows that the $\alpha \rightarrow \omega$ transition starts at $6 \mathrm{GPa}$ for $\mathrm{ZrI}$ and increases up to 11 for ZrIII, which is consistent with Ab initio calculations and the expectation that the presence of interstitial impurities will increase the energy barrier and block the $\alpha \rightarrow \omega$ transformation [6]. The $\alpha \rightarrow \omega$ transition pressure is also influenced by experimental conditions. When experiments were performed under non-hydrostatic conditions, the $\alpha \rightarrow \omega$ transition is observed at $4 \mathrm{GPa}$ for ZrI, a decrease of $2 \mathrm{GPa}$ compared to hydrostatic case. Similar behavior is also observed for both ZrII and ZrIII. This result is not unusual and it is expected that shear deformation will drive this structural phase transition, as previously mentioned.

In case of Ti, we observed the onset of $\alpha \rightarrow \omega$ transition to occur at relatively the same pressure of $\sim 10 \mathrm{GPa}$ for both hydrostatic and nonhydrostatic cases. Existence of $\alpha$ phase under nonhydrostatic conditions to higher pressure could be the result of decreased grain size of our Ti sample. Smaller grain size and therefore increased surfaceto-volume ratio may help increase resistance to shear deformation and help stabilize $\alpha$ phase over a larger pressure range under non-hydrostatic conditions.

As can be seen in Fig. 1 the strongest $\omega$ phase peak, (101/110), appears in the midst of (002) and (101) $\alpha$ phase peaks. This makes it challenging in identifying the onset of $\alpha \rightarrow \omega$ transition when the $(101 / 110) \omega$ phase peak is relatively weak. In order to confirm our results for $\mathrm{Ti}$ studied under non-hydrostatic conditions, we have also performed electrical resistance measurements on the same sample. Electrical resistance measurements are sensitive and can be used to identify electronic changes and disturbances such as atomic shift during structural phase transition. Our measurements show a slight decrease in electrical resistance below $10 \mathrm{GPa}$, which can be associated with thinning and flow of the sample with pressure increase, Fig. 2. In Fig. 2 shown are the results of two measurements on the same $\mathrm{Ti}$ sample, both under non-hydrostatic conditions. At $10 \mathrm{GPa}$ we reach a minimum value followed by an increase in electrical resistance above this pressure. The subsequent increase in resistance above 10 $\mathrm{GPa}$ is consistent with the $\alpha \rightarrow \omega$ transition at this pressure, as was previously established by $\mathrm{ADXD}$ measurements. 


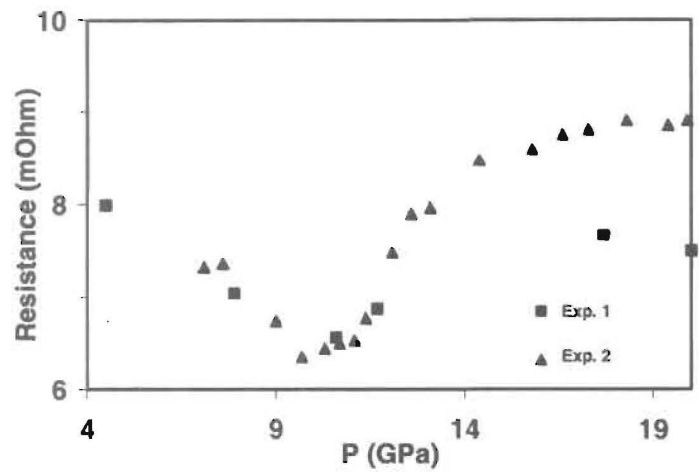

Figure 2. Electrical resistance vs, pressure for $\mathrm{Ti}$ sample.

\section{CONCLUSIONS}

Our experimental results show that there is a clear correlation between increase in pressure at which the $\alpha \rightarrow \omega$ structural phase transition is observed and an increase in interstitial impurity concentration. From the highest purity sample (ZrI) to the lowest purity sample (ZrIII), we see a shift in $\alpha \rightarrow \omega$ transition pressure from $6 \mathrm{GPa}$ to 11 GPa. The transition pressure is also observed to decrease further when $\mathrm{Zr}$ samples are subjected to non-hydrostatic experimental conditions.

However, our experiments also show that nonhydrostatic (shear deformation) effects can be minimized by reducing sample grain size. By performing both $\mathrm{ADXD}$ and electrical resistance measurements on Ti sample with $\sim 100 \mathrm{~nm}$ grain size we were able to determine the onset of $\alpha \rightarrow \omega$ transition. Under both hydrostatic and nonhydrostatic experimental conditions we observe $\alpha \rightarrow \omega$ transition at $10 \mathrm{GPa}$.

Using grain size to help stabilize $\alpha$ phase to higher pressure could prove useful as an alternative to stabilizing $\alpha$ phase by alloying. In some cases alloying may have undesirable affect on other mechanical properties, while grain size reduction can lead to increased material hardness.

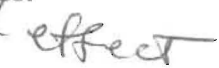

\section{ACKNOWLEDGEMENTS}

LANL is operated by LANS LLC for the DOE and NNSA. The authors acknowledge funding from DOE/NNSA Campaign 1 and 2. Y. K. V. would like to acknowledge support from the National Science Foundation (NSF) under grant No. DMR-0703891. We thank Carnegie-DOE Alliance Center for beamtime and sector 16 scientist for their help with experimental setup. This work was performed at HPCAT (Sector 16), Advanced Photon Source (APS), Argonne National Laboratory. HPCAT is supported by DOE-BES, DOE-NNSA, NSF, and the W.M. Keck Foundation. APS is supported by DOE-BES, under Contract No. DE-AC02-06CH11357. Authors would also like to thank Dr. S. T. Weir (LLNL) for his work on the designer diamond anvil project and Dr. L. Stevens for help with data collection.

\section{REFERENCES}

1. Skriver, H. L., "Crystal Structure From Oneelectron Theory", Phys. Rev. B 31, 1909 (1985).

2. Jameson, J. C., "Crystal Structures of Titanium, Zirconium, and Hafnium at High Pressures", Science 140, 72 (1963).

3. Sikka, S. K., Vohra, Y. K., and Chidambaram, R., "Omega Phase in Materials", Prog. Mater. Sci. 27, 245 (1982).

4. Vohra, Y. K., "Electronic Basis for Omega Phase Stability in Group IV Transition Metals and Alloys", Acta Metallurgica 27, 1671 (1979)

5. Chesnut, G.N., Velisavljevic, N., and Sanchez, L., "Static High Pressure X-Ray Diffractin of Ti$6 \mathrm{~A} 14 \mathrm{~V}$ ", In Shock Compression of Cond. Matter, 2007 (M. Elert, M.D. Furnish, R. Chau, N.C. Holmes, and J. Nguyen, eds.), pp. 27-30,

6. Hennig, R. G., Trinkle, D. R., Bouchet, J., Srinivasan, S. G., Albers, R. C., and Wilkins, J. W., "Impurities Block the $\alpha$ to $\omega$ Martensitic Transformation in Titanium", Nature Materials 4, 129 (2005).

7. Errandonea, D., Meng, Y., Somayazulu, M., and Häussermann, D., "Pressure-Induced $\alpha \rightarrow(1)$ Transition in Titanium Metal: a Systematic Study of Effects of Uniaxial Stress", Physica B 355, I16 (2005).

8. Weir, S. T., Akella, A., Aracne-Ruddle, C., Vohra, Y. K., and Catledge. S. A., "Epitaxial Diamond Encapsulation of Metal Microprobes for High Pressure Experiments", Appl. Phys. Lett. 77, 3400 (2000). 